\title{
Brillouin Spectroscopic Studies of VF2/TFE Copolymers
}

\section{Authors: Zhimin Liu and V. Hugo Schmidt}

This is an Accepted Manuscript of an article published in Ferroelectrics in December 1990, available online: http://www.tandfonline.com/10.1080/00150199008012799.

Liu, Zhimin, and V. Hugo Schmidt. "Brillouin Spectroscopic Studies of VF2/TFE Copolymers."

Ferroelectrics 112, no. 1 (December 1990): 237-243. doi: 10.1080/00150199008012799.

Made available through Montana State University's $\underline{\text { ScholarWorks }}$ scholarworks. montana.edu 


\title{
BRILLOUIN SPECTROSCOPIC STUDIES OF $\mathrm{VF}_{2}$ /TFE COPOLYMERS
}

\author{
ZHIMIN LIU and V. HUGO SCHMIDT \\ Department of Physics, Montana State University, Bozemann, MT 59717 USA
}

(Received May 25, 1990)

The temperature dependence of sound velocity was studied in thin sheets of isotropic and oriented copolymers of vinylidene fluoride ( $81 \mathrm{~mol} \%)$ and tetrafluoroethylene ( $19 \mathrm{~mol} \%)$ by Brillouin scattering. For oriented samples, we studied the differences between the sound velocities along the $x$-axis (chain direction) and $y$-axis (perpendicular to $x$ in sample plane). The ferroelectric-paraclectric transition points were not observed because this $T_{r}$ is very close to the melting point of these copolymers. A partial thermal hysteresis loop was observed for all samples. The transitions from paraclectric phase to ferroelectric phase are completed near $85^{\circ} \mathrm{C}$ for the isotropic sample and the oriented sample with sound propagating along $y$. For propagation along $x$-axis, this transiton is completed at about $100^{\circ} \mathrm{C}$ which is very close to results from other methods. The behaviors of temperature dependence of sound velocity along $x$ and $y$ axes are quite different, as we observed also in $\mathrm{VF}_{2} / \mathrm{TrFE}$ copolymers. It allows generalization of our conclusions concerning copolymers of vinylidene fluoride and trifluoroethylene.

\section{INTRODUCTION}

The polymer vinylidene fluoride $\left(\mathrm{PVF}_{2}\right)$ and its copolymers with trifluoroethylene (TrFE) and tetrafluoroethylene (TFE) are ferroelectric. ${ }^{1-3}$ A copolymer of vinylidene fluoride and tetrafluoroethylene (hereafter abbreviated $\mathrm{VF}_{2} / \mathrm{TFE}$ ) prepared under any crystallization conditions, possesses the form I ( $\beta$ phase) crystal only, while the form I crystal of $\mathrm{VF}_{2}$ can be obtained from the form II crystal by stretching a film or by applying a very high electric field. ${ }^{4}$ The ferroelectric to paraelectric (FE-PE) phase transition was never observed in $\mathrm{PVF}_{2}$ because it may be obscured by melting temperature which caused an argument concerning whether $\mathrm{PVF}_{2}$ is ferroelectric or not. The FE-PE transition has been commonly observed in copolymers with TrFE, but the introduction of the new chemical species - $\mathrm{CHF}$ - created suspicion that the CHF group might cause this transition. Copolymer of TFE are of particular interest because they do not introduce this new chemical species $-\mathrm{CHF}$ - or tacticity defects as does TrFE, yet their FE-PE phase transitions have been detected. ${ }^{5}$ They can be viewed simply as $\mathrm{VF}_{2}$ containing an increased number of head-to-head $\left(\mathrm{CF}_{2}-\mathrm{CF}_{2}-\right)$ defects. ${ }^{6}$ Lovinger ${ }^{1}$ indicated that the FE-PE transition point is so close to melting point that no transition can be observed. Later, Lovinger et al. detected the Curie transition beginning at about $122^{\circ} \mathrm{C}$ in a differently prepared sample. ${ }^{5}$

Any transitions occurring in the amorphous or crystalline regions in general affect the elastic properties and can therefore be investigated by Brillouin scattering. Krüger et al. have studied 70/30 $\mathrm{VF}_{2} \mathrm{TrFE}$ copolymers by Brillouin scattering. ${ }^{7}$ They have detected the FE-PE phase transitions by analyzing kink, between segments with different slopes in the sound velocity $v s$. temperature curves. Also they observed obvious hysteresis between heating and cooling runs. In this paper we 
present the temperature dependence of sound velocity for an isotropic sample and for a stretched sample with sound propagating along the $x$ axis (chain direction) and the $y$ axis (perpendicular to $x$ axis in sample plane). We observed glass transitions and partial FE-PE phase transitions by the same analysis method as Krüger used. The purpose of this work is to compare the different transition behaviors along the chain (intramolecular) direction and along the intermolecular direction. We previously got different transition temperatures from the temperature dependence of sound velocity along the $x$ and $y$ axes for $\mathrm{VF}_{2} / \mathrm{TrFE}$ copolymers with various compositions. The present studies of $\mathrm{VF}_{2} / \mathrm{TFE}$ will clarify whether that difference is general for $\mathrm{VF}_{2}$ copolymers or whether it results from introduction of TrFE.

\section{EXPERIMENT}

Copolymer samples were prepared from Kynar 7201, a random copolymer of $\mathrm{VF}_{2} /$ TFE with 81/19 molar ratio in powder form manufactured by Pennwalt Corporation. Light scattering experiments need a transparent film as sample. We prepared this film by the following procedure. First we put the copolymer powder between two pieces of copper with a special plastic film of melting point near $400^{\circ} \mathrm{C}$ between the copper and powder. The pieces of copper were compressed together by a strong spring. Then we heated the whole fixture to $180^{\circ} \mathrm{C}$ for several minutes and quenched it in $0^{\circ} \mathrm{C}$ water. Because the time above melting temperature was not long enough, the powder was melted but had not formed a uniform film. Then we reheated the fixture to $180^{\circ} \mathrm{C}$ and kept it at this temperature for 15 minutes and quenched it to $0^{\circ} \mathrm{C}$ to obtain a transparent film. We found that the reheat procedure is necessary to obtain samples with smooth surface and correspondingly good optical properties. A single heating cycle only creates an opaque or translucent film. The samples prepared by this quenching method have melting point of $126^{\circ} \mathrm{C} .{ }^{8}$ Films thus obtained were stretched by a ratio of $3.5: 1$ at $65^{\circ} \mathrm{C}$, then annealed at $100^{\circ} \mathrm{C}$ for 24 hours. Annealed samples were cooled naturally under tension. We measured the temperature dependence of sound velocity for three samples. One is isotropic and the other two are stretched samples which were used for measurement of sound velocity along the $x$ and $y$ axes respectively. In the following discussion, we denote them and their transition points by subscripts $i, x, y\left(T_{g i}\right.$. for glass transition and $T_{c i}$.. for FE-PE transition).

The sample was placed in an optical cell described in a previous paper. ${ }^{9}$ The temperature of the optical cell was controlled within $\pm 0.2^{\circ} \mathrm{C}$. A Lexel model 95 2 argon-ion laser operating in a single mode at wavelength $5145 \AA$ was used as a light source. The frequency shifts were measured by a piezoelectrically scanned Burleigh 5-pass Febry-Perot (F-P) interferometer. The sound velocity $V$ was related to the frequency shift $F_{B}$ by ${ }^{10}$

$$
V=\lambda F_{B} / \sqrt{2},
$$

in the case of $90^{\circ}$ scattering geometry, where $\lambda$ is the laser wavelength. We used one sample for both heating and cooling runs. First we cooled the sample to about $-65^{\circ} \mathrm{C}$ without measurement, then heat it with heating rate of about $0.5 \%$ minute. 
The temperature steps varied between $1.5^{\circ}$ and $2.5^{\circ}$. Each step lasts about 25 minutes for a number of scans by the F-P interferometer. When the temperature is just close to copolymer melting point at about $120^{\circ} \mathrm{C}$, the sample becomes soft which can be observed from the optical windows and from the degraded Brillouin spectrum. We held this temperature for about 10 minutes, then reduced temperature to start the cooling run.

\section{RESULTS AND DISCUSSION}

Figure 1a-c shows the temperature dependence of velocity of sound propagating along the $x$ (Figure 1a) and $y$ (Figure 1b) axes for stretched samples, and for an isotropic sample (Figure 1c), in the temperature region from $-65^{\circ}$ to $40^{\circ} \mathrm{C}$ where the glass transitions occur. We fit these data to the equation ${ }^{11}$

$$
V(T)=a_{1}-a_{2} T-a_{3}\left[\operatorname{abs}\left(T-T_{g}\right)\right],
$$

which describes sound velocity up to room temperature. The fitting procedure determined the glass transitions $T_{g}$ which concern freezing out of motions in the amorphous part of the copolymer. The parameters of $a_{i}=(i=1,2,3)$ describe the slopes of sound velocity vs. temperature curves in this temperature region. The $T_{g x}=-45^{\circ} \mathrm{C}$ and $T_{g y}=-47^{\circ} \mathrm{C}$ values for the stretched sample are very close to the values near $-50^{\circ} \mathrm{C}$ obtained by other methods. ${ }^{5,8}$ They are almost the same because these transitions correspond to the same physical process in similar samples. Significant differences were found in $T_{c x}$ and $T_{c y}$ for similar samples, which will be discussed later in detail. The value $T_{g i}=-40^{\circ} \mathrm{C}$ is a little higher than $T_{g s}$ and $T_{g y}$. The stretched (oriented) copolymers have more orderly structure than isotropic ones and may have lower glass transition. The rates of decrease of sound speed $v s$. temperature in copolymers of $\mathrm{VF}_{2} / \mathrm{TFE}$ are $5.5 \mathrm{~m} / \mathrm{s}$ per degree along $x, 3.5$ $\mathrm{m} / \mathrm{s}$ per degree along $y$ and $3.75 \mathrm{~m} / \mathrm{s}$ per degree for the isotropic sample. The decrease rate for the isotropic sample is between those along $x$ and $y$ because the crystal cells take random directions. These results are similar to those of $4.6 \mathrm{~m} / \mathrm{s}$, $2.1 \mathrm{~m} / \mathrm{s}$ and $2.9 \mathrm{~m} / \mathrm{s}$ per degree in $80 / 20 \mathrm{VF}_{2} / \mathrm{TrFE}$ copolymers. ${ }^{12}$

Figures 2-4 show temperature dependence of sound velocity up to about $120^{\circ} \mathrm{C}$ for the above-mentioned three samples. Determining the FE-PE phase transitions is very difficult by Brillouin scattering because this transition beginning at $122^{\circ} \mathrm{C}$ is thwarted by the onset of melting for quenched samples with melting temperature $T_{m}=126^{\circ} \mathrm{C} .{ }^{5}$ In the studies of this copolymer by Brillouin scattering, we identified FE-PE phase transitions by detecting kinks between basically linear parts with different slope of sound velocity vs. temperature curves. ${ }^{7}$ It is difficult to observe the completed phase transition in these samples by Brillouin scattering. In our experiment, the samples suffered premelting effects at temperatures above $110^{\circ} \mathrm{C}$ and the Brillouin spectra become so bad above $120^{\circ} \mathrm{C}$ that the data seem untrustworthy. We have not observed a completed phase transition but have seen some clues of this transition. In Figures 2 and 3, the temperature dependence of sound velocity along $y$ and in the isotropic sample show a clear tendency toward finishing the transitions, which appear likely to be completed not very above from the temperature where we stopped. We have determined that the paraclectric to fer- 


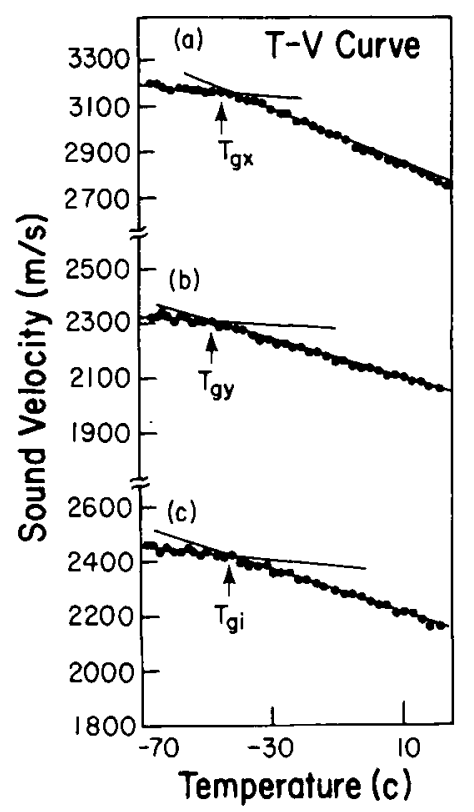

FIGURE 1 Temperature dependence of sound velocity for $81 / 19 \% \mathrm{VF}_{2} / \mathrm{TFE}$ copolymers in temperature range of $-65^{\circ}$ to $23^{\circ} \mathrm{C}$ (a) stretched sample for sound propagation along $x$ axis, (b) stretched sample for sound propagation along $y$ axis, (c) isotropic sample.

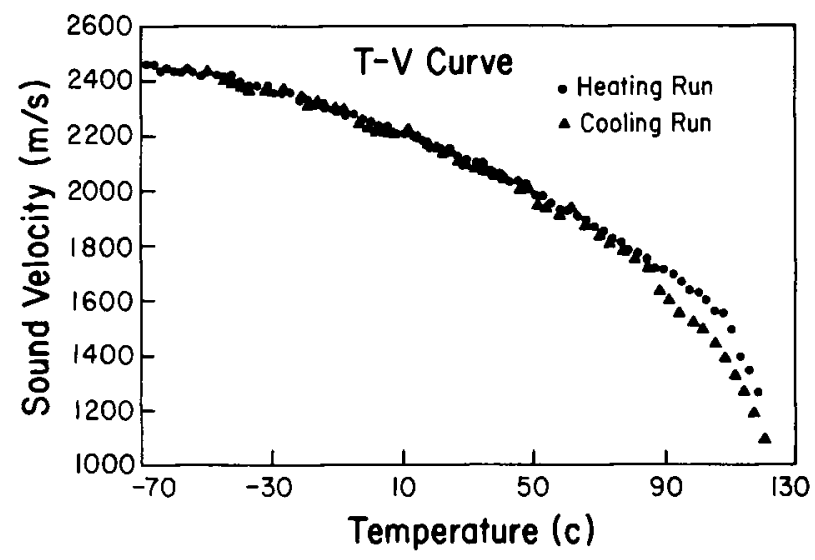

FIGURE 2 Sound velocity for isotropic sample, $O$ : heating run, $\triangle$ : cooling run.

roelectric phase transition is completed at about $85^{\circ} \mathrm{C}$ upon cooling and have seen obvious hysteresis.

In Figure 4 showing the velocity of sound propagating along $x$, we could not see any tendency toward completing the FE-PE transition near our highest temperature. We consider the junction point where the cooling run coincide with heating run as the para/ferro transition $T_{c}^{\prime}$, at about $100^{\circ} \mathrm{C}$ which is close to Lovinger's result of $95^{\circ} \mathrm{C}^{1}$ and $110^{\circ}-115^{\circ} .5$ Comparing Figure 4 with Figures 2 and 3 , we conclude that the transition behaviors of this copolymer along $x$ and $y$ axes are quite different even though the transition points have not been determined accu- 


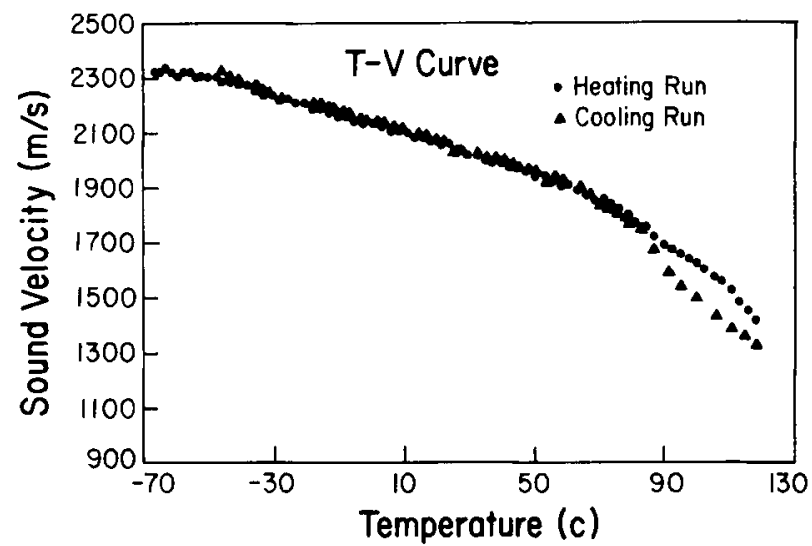

FIGURE 3 Sound velocity for stretched sample for sound wave propagation along $y$ axis. $\bigcirc$ : heating run, $\triangle$ : cooling run.

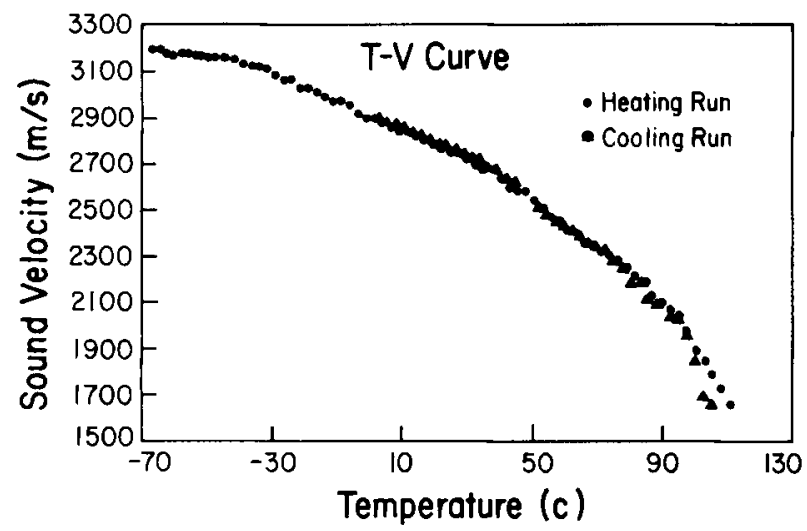

FIGURE 4 Sound velocity for stretched sample for sound wave propagation along $x$ axis. $O$; heating run, $\triangle$ : cooling run.

rately. We see that $T_{c x}^{\prime}$ is not equal to $T_{c y}^{\prime}$, and can infer that $T_{c x}$ is not equal to $T_{c y}$ from the Figures.

The difference between $T_{c x}$ and $T_{c y}$ in a similar sample is not a new discovery. Krüger et al. ${ }^{7}$ found $20^{\circ} \mathrm{C}$ difference between $T_{c x}$ and $T_{c y}$ in their Brillouin scattering studies for $70 / 30 \mathrm{~mol}_{0} \mathrm{VF}_{2} / \mathrm{TrFE}$ copolymers. They thought that $T_{c x}$ and $T_{\mathrm{cy}}$ correspond to the same physical process and explained this difference as an annealing effect, but this conclusion is open to question because the samples they used in measurements along $x$ and $y$ were prepared with different annealing times. We found the same thing happened for copolymers of $\mathrm{VF}_{2} / \mathrm{TrFE}$ with various compositions from $80 / 20$, to $60 / 40 \mathrm{~mol} \% .^{12}$ The $x$ and $y$ samples used in our measurements were cut from the same piece so they have the same annealing history. The difference between $T_{c x}$ and $T_{c y}$ can not be explained as an annealing effect. We believe that this difference means that $T_{c y}$ and $T_{c x}$ represent total different physical processes or transitions. This difference is also reflected in the para/ferro 
phase transitions. As mentioned above, $T_{c y}^{\prime}$ is about $85^{\circ} \mathrm{C}$ and $T_{c .}^{\prime}$ is about $100^{\circ} \mathrm{C}$

Brillouin scattering has the unique characteristic that allows one to study the properties related to the special direction we are interested in. The sound velocity along $y$ axis mainly reflects the change of intermolecular van der waals bonding. The sound velocity along $x$ axis mainly reflects the change of intramolecular, $C-C$ bonds. There is no reason to presume $T_{c x}$ and $T_{c y}$ should be the same. The FE$\mathrm{PE}$ phase transition in ferroelectric copolymers is caused by intramolecular change from the all-trans conformation to irregular TGT $\bar{G}$ conformations. So the $T_{c x}$ in Brillouin scattering should correspond to the well-known Curie temperature found in other measurements. $T_{r y}$ corresponds to a transition in the intermolecular direction, and may reflect a discrete structural change in the ferroelectric phase.

We review, for comparison with our $\mathrm{VF}_{2} / \mathrm{TFE}$ copolymer data, other results indicating two transitions which, however, are all for $70 / 30 \mathrm{VF}_{2} / \mathrm{TrFE}$ copolymers. Faria and Latour ${ }^{13}$ made thermally stimulated depolarization (TSD) measurements on poled but unstretched samples with applied mechanical vibration which gave superimposed electrical noise from the piezoelectric effect but only when the sample was in a piezoelectric (probably ferroelectric) phase. They found two peaks upon heating, first an " $A$ " peak near $100^{\circ} \mathrm{C}$ which they attributed to a ferro-ferroelectric transition of unspecificd nature, and then a sharper " $B$ " peak at $108^{\circ} \mathrm{C}$ where the piezoelectric noise ceased, which they consider to be the ferro-paraclectric transition. Delzenne ${ }^{14}$ reported that the ferro-paraelectric transition takes place in two stages, or that perhaps there are two transitions. From these results, we conclude that the process of transition from ferro ito paraelectric phase is unclcar.

According to our data and the above review, we believe that the supposedly existing two transitions are transitions in the $\mathrm{C}-\mathrm{C}$ bond direction and in the interchain direction which happen at different temperatures. We expect that the ferro/para transition will not be observed in the $y$-axis sound velocity because the intermolecular bonding in that direction is similar for all-trans and helical conformations for stretched but unpoled samples. So we identify $T_{c y}$ to be a structural transition point which has totally different physical significance from $T_{c x}$. By similar reasoning, if there is a structural transition in the $x(\mathrm{C}-\mathrm{C}$ bond $)$ direction, sound waves propagating along $y$ will not see it. Only $T_{c}\left(T_{c x}\right)$ which corresponds to the transition from all trans to disordered helical conformations is observed because this sound wave is only sensitive to change in the $x$ direction, intramolecular change.

We attribute the similar transition behavior for $\mathrm{VF}_{2} / \mathrm{TFE}$ copolymers to similar phenomena as in $\mathrm{VF}_{2} / \mathrm{TrFE}$ even though the ferro/para transitions have not been observed completely. Our results for $\mathrm{VF}_{2} / \mathrm{TFE}$ allow generalization of our conclusions about two transitions obtained from the copolymers of $\mathrm{VF}_{2} / \mathrm{TrFE}$ and exclude any possible effect of the atomic group $\mathrm{CHF}$ found only in $\mathrm{VF}_{2} / \mathrm{TrFE}$ copolymers. Because $\mathrm{VF}_{2}$ /TFE copolymer is equivalent to $\mathrm{VF}_{2}$ containing a higher percentage of head-to-head defects, it is a reasonable inference that two-transition behavior should be possible in $\mathrm{PVF}_{2}$ homopolymer, to the extent that it is not obscured by melting.

\section{ACKNOWLEDGEMENTS}

This work was supported by Department of Energy Grant No. DE-FG06-87ER4.5292. 


\section{REFERENCES}

1. A. J. Lovinger, Macromolecules, 16, 1529 (1983).

2. A. J. Lovinger, G. T. Davis, T. Furukawa and M. G. Broadhurst, Macromolecules, 15, 323 (1982).

3. J. C. Hilks, T. E., Jones and J. C. Logan, J. Appl. Phys., 49, 6092 (1978).

4. G. T. Davis, J. E. McKinney, M. G. Broadhurst and S. C. Roth, J. Appl. Phys., 49, 4998 (1978).

5. A. J. Lovinger, G. E. Johnson, H. E. Bair and E. W. Anderson, J. Appl. Phys., 56, 2412 (1984).

6. W. W. Doll and J B. Lando, J. Macromol. Sci. Phys. Ed., B4, 897 (1970).

7. J. K. Krügcr, J. Petzelt and J. F. Legrand, Colloid \& Polymer Science, 264, 791 (1986).

8. S. Tasaka and S. Miyata, J. Appl. Phys., 57, 906 (1985).

9. M. Yamada and V. H. Schmidt, Rev. Sci. Instrum., 49, 1226 (1978).

10. C. H. Wang. D. B. Cavanaugh and Y. Higashigaki, J. Polym. Sci. Polym. Phys. Ed., 19, 941 (1981).

11. J. K. Krüger, R. Roberts, H. G. Unruh, K. P. Fruhauf, J. Helwig and H. E. Müser, Prog. Colloid \& Polymer Sci., 71, 77 (1985).

12. Z. Liu and V. H. Schmidt, unpublished.

13. R. M. Faria and M. Latour. J. Phys. France, 49, 2089 (1988).

14. P. Delzenne, Ph.D. Thesis, Univ. of Grenoble, France (1986). 